\title{
Design and Study of Chirped Fiber Bragg Grating for Sensing of Hazardous Gases
}

\author{
Dr. Anubhuti Khare \\ Department of Electronics and Communication \\ University Institute of Technology, \\ Rajeev Gandhi Technical University, \\ Bhopal M.P. India
}

\begin{abstract}
This paper deal with the application of Chirped Fiber Bragg Grating in sensing of hazardous gases. By developing a mathematical model for the CFBG showing the effect of temperature on parameters of grating. Due to immunity of electrical magnetic interference, high sensitivity, compactness and simple to fabrication we preferred CFBG for sensing purpose. By measuring the reflected spectrum and fitting with mathematical model based on Transfer Matrix Method, the effect of temperature on CFBG have been studied. Simulation results are taken for change in spectrum due to rises in temperature due to reaction of hazardous gases with coated chemical.
\end{abstract}

Keywords: Temperature sensor, chirped fibre Bragg grating, fibre optic sensors

\section{INTRODUCTION}

A CFBG is a grating having a-periodic pitch and can be in Sensing and telecommunication. There are three important parameters of CFBG by which we can define properties like Grating periods, refractive index and length of gratings. According to figure 1,As it reflect the incident light with particularly predetermine wavelength while passing all other at the same time. As the wavelength varies with temperature the detection of wavelength will yield information about these quantities. The sensor based on

CFBG have more advantage like light weight, small size and high temperature sensing devices. This research seek to develop new approach to improve the performance of FBG throgh Chirping of Optical Fiber Bragg Grating.According to figure no. 1 the period of grating is increasing with distance.

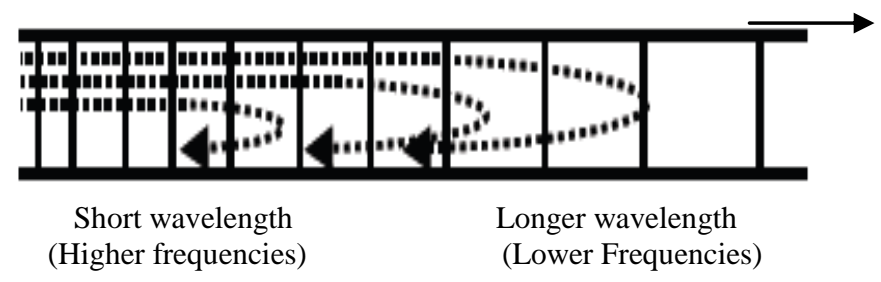

Fig.1

\author{
Jaikaran Singh \\ Department of Electronics and Communication \\ University Institute of Technology, \\ Rajeev Gandhi Technical University, \\ Bhopal M.P. India
}

\section{THEORY}

According to given equation No. 1 the length of CFBG is the function of varied period as

$$
L(z)=\Lambda_{0}+\Lambda_{1} z
$$

$\mathrm{Z}$ is the value of position having the value from 0 to 0.01

The value of reflection coefficient vary with the value of $\mathrm{z}$ at different points along its length. According to equation no. 2 the refractive index of CFBG can be expressed as

$$
n(z)=n(z)+8 n \cos \left(2 \pi \frac{z}{\Lambda}\right)+2 \int_{0}^{z} \phi(\xi) d(\xi)
$$

Where $A$ is the grating period and $\varphi(\zeta)$ is the phase of chirped grating. And in given equation the refractive index $n(z)$ is the function of $z, \Lambda$. For simulation result we are using Direct Integration Matrix methods. The periods change along $\mathrm{z}-$ direction so that the value of Bragg wavelength vary with $z$.since the $\delta$ n along $z$-direction has the same effect as changing the period along the $\mathrm{z}$-direction. The chirped grating can be modeled by the chirped parameter

$$
\begin{aligned}
& F=\frac{(F W H M)^{2}}{z^{2}} \phi(z) \\
& F=-4 \pi n_{E D} \frac{(F W H M)^{2}}{\lambda_{D}^{2}} \frac{d \lambda_{D}}{d z}
\end{aligned}
$$

Where $\mathrm{F}$ is the factor of fractional change over entire length and FWHM is full width ay half maximum of grating profile.According to TMM the mathematical formula for the $\mathrm{F}$ is given as

This figure show that the linear chirp Bragg Grating, 


$$
F=\left[\begin{array}{cc}
\cosh \left(R_{B} \times \frac{L}{N}\right)-\left(\frac{\sigma}{R_{B}}\right) \times \sinh \left(R_{B} \times \frac{L}{N}\right) \times 1 i & -\left(\frac{K_{u c}}{R_{B}}\right) \times \sinh \left(R_{B} \times \frac{L}{N}\right) \times 1 i \\
\left(\frac{K_{u C}}{R_{B}}\right) \times \sinh \left(R_{B} \times \frac{L}{N}\right) \times 1 i & \cosh \left(R_{B} \times \frac{L}{N}\right)+\left(\frac{\sigma}{R_{B}}\right) \times \sinh \left(R_{B} \times \frac{L}{N}\right) \times 1 i
\end{array}\right]
$$

where

$$
\begin{aligned}
& \sigma=2 \times \pi \times n_{\mathrm{etf}} \times\left(\frac{1}{\lambda(k)}-\frac{1}{\lambda_{D}}\right)+2 \times \pi \times \frac{\delta n_{\mathrm{eff}}(z)}{\lambda(k)}+\left(4 \times \pi \times n_{\mathrm{eft}}\right) \times c \times\left(2 \times\left(n_{\mathrm{tg}}+\delta n_{\mathrm{eft}}\right)\right) \times \frac{-L \frac{1}{2}+n \times L \frac{1}{N}}{\left(\lambda_{D}\right)^{2}} \\
& \lambda_{D}=1550 \times 10^{-9}-L 1 \times n_{c t} \times c+2 \times n_{e t t} \times c \times\left(\frac{L 1 \times n}{N}-\frac{0.5 \times L 1}{N}\right) \\
& k_{a c}=\frac{\pi \times \delta_{n e s}(z)}{\lambda(k)} \quad R_{B}=\sqrt{K_{a c}^{2}-\sigma^{2}}+\varepsilon \\
& R(k)=\left(a b s\left(\frac{F(2,1)}{F(1,1)+\pi}\right)\right)^{2} \quad z=\frac{F(2,1)}{F(1,1)+\pi}
\end{aligned}
$$

\section{FABRICATION}

Anbhawa Nand et al(2007)[1]. We have different type of method for fabrication of CFBG for application of sensing purpose. It can be designed with two algorithms like amplitude or periodic chirping. In amplitude of the refractive index varies along the length and periods keep constant and other method in which we varies the period of grating along the length and we keep constant amplitude of refractive index. Periodic chirped grating have special feature and usable in sensing purpose. The method for fabrication of OFBG is is chirped on phase mask Technique.

It is the controlled method of fabrication of CFBG. The mask is divided into subsection having own period change with length of grating. We fabricate the $\mathrm{N}$ section with different period. However, certain characteristic properties of periodicity chirping such as the potential to create a broader bandwidth and its relative ease of fabrication, offer advantages in specific applications and thus have been used extensively. Periodicity chirping can be introduced by: using a chirped phase mask, subjecting the fibre to a strain gradient during fabrication, writing gratings on prestrained fibres, straining the fibre after grating fabrication, writing gratings in fibre tapers, or interfering wavefronts of dissimilar curvatures in a holographic arrangement. A schematic diagram of a chirped grating employing periodicity chirping is illustrated in figure 1 .

\section{EFFECT OF TEMPERATURE ON CFBG:}

The effect of temperature on parameters of CFBG are shown with the below equation. The basic principal work on measure the change in wavelength due to temperature increase as

$$
\lambda_{B}(z)=2 n_{e q i}(z) \Lambda(z)
$$

Where $\lambda_{\mathrm{B}}(\mathrm{z})$ is Bragg wavelength, $\mathrm{n}_{\mathrm{eff}}$ and $\Lambda z^{u}$ are the

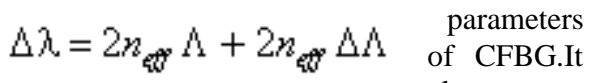

also vary

the value of incident wavelength through equation due to effect of increase the temperature

The shift in Bragg wavelength change due to $\mathrm{n}_{\text {eff }}$ and bragg period.The light from RF source incident on CFBG and some part reflected due to presence of grating. The change in reflected spectrum can be analysis for showing the effect of increase temperature. The effect of Temperature on $\mathrm{z}$ is given as

And

$$
z(T)=z(1+\alpha \Delta T)
$$

$$
A(T)=\Lambda(1+\alpha \Delta T)
$$

Where is thermo coefficient factor having value of $\alpha \approx-10^{-7}$

\section{RESULTS}

According to given below figure the refecltion spectrum increase in start and become constant and again it decrease at $1551 \times 10^{-6}$ The figure shown for the length of $0.01 \mathrm{~cm}$

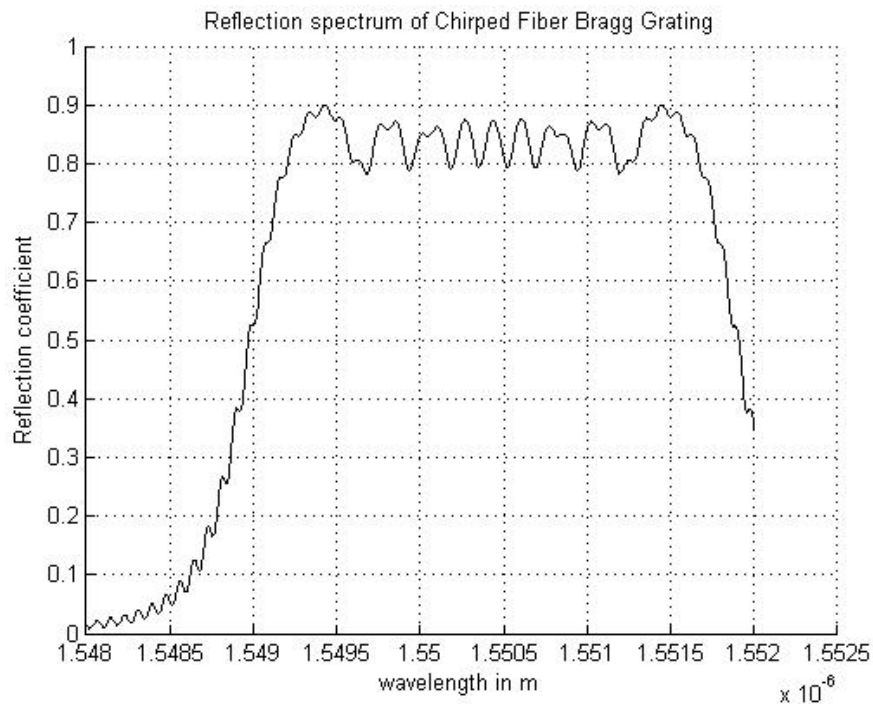

Fig.2

According the figure 2 , when we reduce the length of grating the Chirped Fiber Bragg Grating work as a uniform grating. 


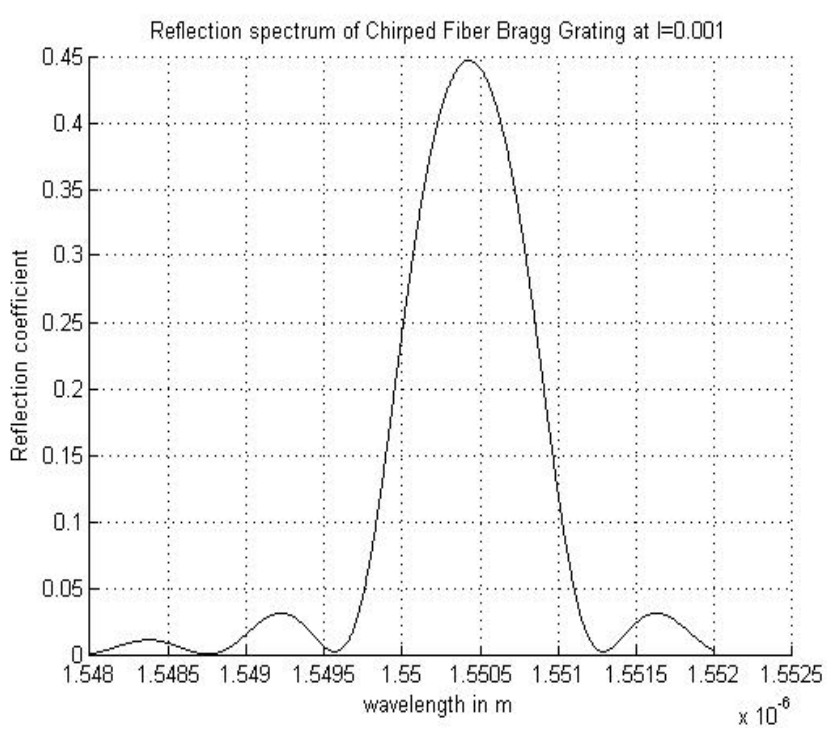

Fig.3

According to figure below the FWHM is decreasing as we increasing the length of CFBG.It also decrease the amplitude of reflection parametsr.

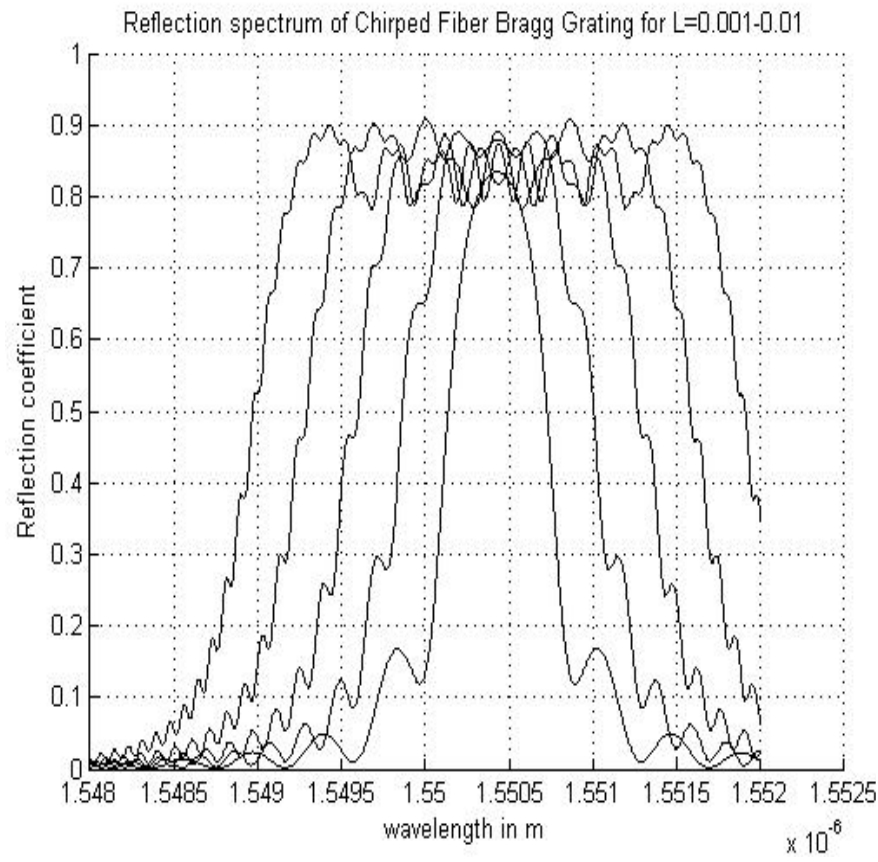

Fig.4

The below figure show the effect of temperature on temperature, the the value of reflection coefficient id decreasing with temperature.

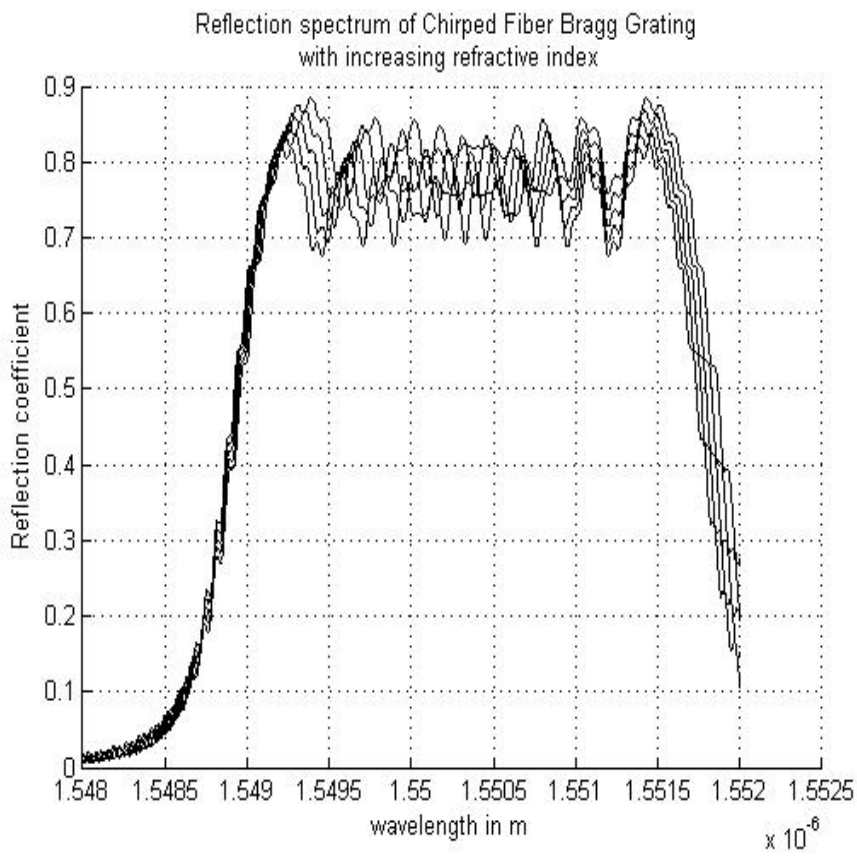

Fig.5

The given graph show the effect of tempearure on delta neff which is the important property of CFBG.

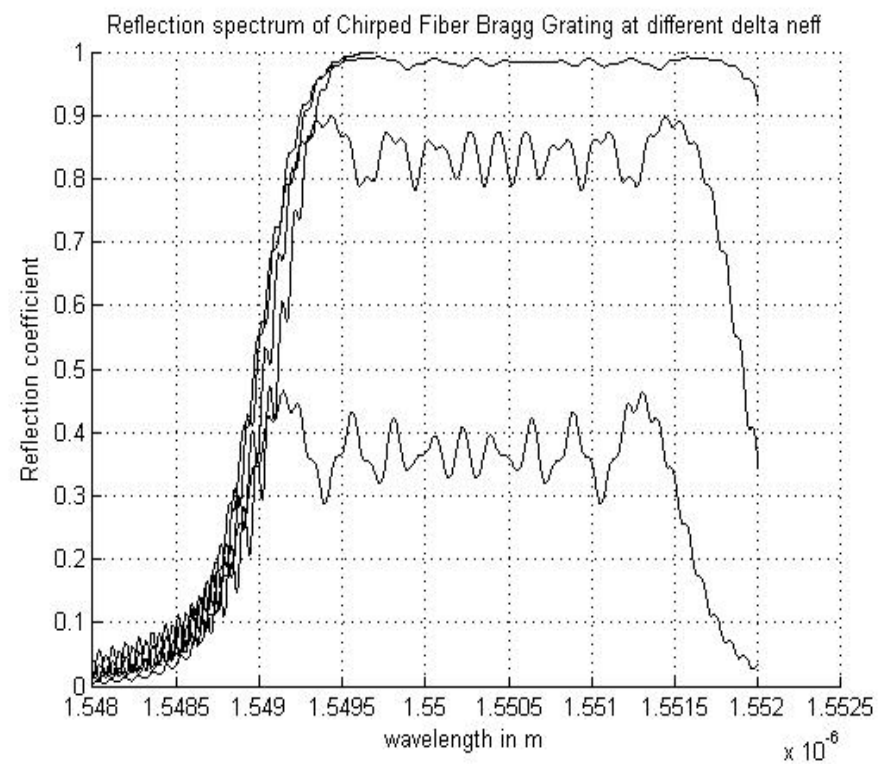

Fig.6

This figure show that the amplitude of reflection coefficient increase as the temperature increase with hazardous gas .

\subsection{Analysis}

The Parameter of CFBG are varying with temperature.and we saw the effect of Temperature on CFBG and results are shown in figure in above figures. 


\subsection{Application to Hazardous Gas Sensing}

Since there are many gases in environment which are main reason of global warming.Out of which Methane is a major cause of global warming by producing heat through reaction with oxygen. The concentration of methane is increasing regularly by which the tempaeruture of environment is increasing throght the reaction

$$
\mathrm{CH}_{4}(\mathrm{~g})+2 \mathrm{O}_{2}(\mathrm{~g}) \rightarrow \mathrm{CO}_{2}(\mathrm{~g})+2 \mathrm{H}_{2} \mathrm{O}(\mathrm{l})+891 \underline{\mathrm{kJ}} / \underline{\mathrm{mol}}
$$

Due to combustion, $\mathrm{CO}_{2}$ generate and oxygen depletes. which causes suffocation to living being. If the concentration of methane increases in such a manner then temperature of environment rises and deficiency of oxygen occurs[7].

\section{CONCLUSION}

This paper presented for the use of CFBG for sensing of hazardous gas like methane.We developed a algorithm for the design of CFBG with the method of Transfer Matrix Method.This methos is very important for design of any CFBG.

\section{REFERENCES}

[1] Basir Ahmed Tahir*, Jalil Ali, Rosly Abdul Rahman"Fabrication of fiber grating by phase mask and its sensing application", Journal of Optoelectronics and Advanced Material Vol. 8, No. 4, August 2006, p. 1604 1609
[2] Naum K. Berger *, Boris Levit, Baruch Fischer," Complete characterization of optical pulses using a chirped fiber Bragg grating, Optics Communications 251 (2005) 315321

[3] Chin-Hua Wang, Lawrence R. Chen, and Peter W. E. Smith," Analysis of chirped-sampled and sampled-chirped fiber Bragg gratings", APPLIED OPTICS _ Vol. 41, No. 9 _ 20 March 2002 1654-1660

[4] Cheng-cheng Sun*, Chun-yun Li, Xiao-ning Yu,"Study on sampled chirped fiber gratings", Optics Communications 218 (2003) 297-302

[5] Seung-Hyun Cho, Jaedong Park, Byoungwhi Kim, and Min-Ho Kang," Fabrication and Analysis of Chirped Fiber Bragg Gratings by Thermal Diffusion", ETRI Journal, Volume 26, Number 4, August 2004,371-374

[6] Jaw-Luen TANG1 and Jian-Neng WANG2," Simultaneous Temperature and Strain Sensing with Optical Fiber Bragg Gratings", Sensors \& Transducers Magazine ( $\& \& T e$ Digest), Vol.68, Issue 6, June 2006, pp.597-605

[7] J. Canning, Invited Review, "Fibre Gratings and Devices for Sensors and Lasers" Lasers and Photonics Reviews, Wiley, USA, 2008 